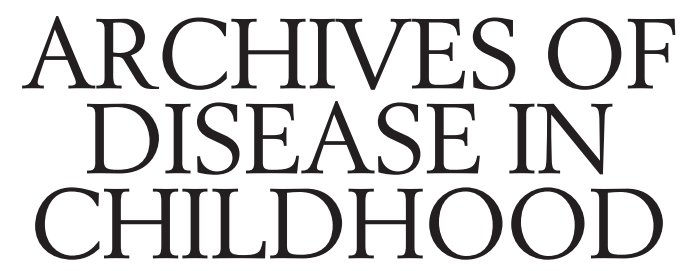

The Fournal of the Royal College of Paediatrics and Child Health

\title{
Annotations
}

\section{Paediatric HIV infection}

Globally paediatric HIV infection represents a major setback to child health. The World Health Organisation estimates that over 20 million adults and 1.5 million children have been infected with HIV since the pandemic began 20 years ago. World wide over a thousand children a day are born with HIV. The highest incidence rates are in sub-Saharan Africa and South Asia, particularly now in urban India. ${ }^{1}$ The Day report recently published projections for the incidence and prevalence of AIDS in England and Wales for 1995-9. Although a fall is expected in new AIDS cases among homo/bisexual males, a $25 \%$ rise in the heterosexual acquisition category, and a $60 \%$ increase in the incidence of AIDS in children of HIV infected mothers are projected. ${ }^{2}$ Absolute numbers of known infected children are still small in the UK, with 380 vertically infected children reported by October 1996 (table 1). Around $80 \%$ of the children with confirmed HIV infection reside in the Thames regions (predominantly in London) with about $7 \%$ resident in Scotland.

The most exciting advance in preventing paediatric HIV infection for countries that can afford it, has been the report from the AIDS Clinical Trials Group (ACTG) protocol 076 that zidovudine (AZT) given perinatally to a selected group of HIV infected pregnant women and their infants reduced the risk of vertical transmission by two thirds $(25 \%$ to $8 \%){ }^{3}$ The mothers received AZT in the second and third trimester, followed by intrapartum intravenous AZT, and AZT was given to infants for the first six weeks of life. Vertical transmission rates vary world wide, with reports from prospective studies ranging from $15-20 \%$ in Europe to $25-35 \%$ in Africa. ${ }^{45}$ An increased rate of transmission is seen in premature infants, the first born of twins, and in women with more advanced HIV disease with lower CD4 counts and higher plasma HIV RNA

Table 1 Infection status of children born to HIV positive mothers reported to the British Paediatric Surveillance Unit or Royal College of Obstetricians and Gynaecologists by 31 October 1996 (from Newsletter 29)

\begin{tabular}{lllll}
\hline & AIDS & $\begin{array}{l}\text { Other } \\
\text { infected }\end{array}$ & Indeterminate & Uninfected \\
\hline Thames & 162 & 131 & 224 & 138 \\
$\begin{array}{l}\text { Rest of England, Wales, } \\
\quad \text { Northern Ireland }\end{array}$ & 38 & 22 & 50 & 42 \\
$\begin{array}{l}\text { Scotland } \\
\text { Total }\end{array}$ & 14 & 13 & 35 & 110 \\
\hline
\end{tabular}

concentrations. ${ }^{67}$ Although retrospective analyses have suggested a slightly increased transmission rate in vaginal compared with caesarean section delivery, results are still awaited from randomised prospective trials of mode of delivery and vaginal viricides. The additional risk of transmitting HIV by breast feeding has been estimated to be $7-22 \%$, suggesting that where bottle feeding is a safe and culturally acceptable option it should be encouraged for infants born to HIV positive mothers. ${ }^{8}$

The opportunity of substantially reducing vertical transmission and improved care for known infected infants now means that the case for offering HIV testing to pregnant women in high prevalence areas is very strong, as recommended by the Department of Health since 1992. In 1994 the unlinked anonymous HIV seroprevalence monitoring programme demonstrated that one in 360 pregnant women was infected in Inner London. This compared with one in 10000 in England and Wales outside the south east. ${ }^{9}$ In London, with almost 200 HIV positive women delivering each year, a number of hospitals have developed active programmes offering universal named antenatal HIV testing, but uptake rates in many maternity units remain low. The estimated costs of preventing HIV infection in a child are high, but lifetime expenditure on medical and social care of infected children will probably be higher. ${ }^{10}$ In London the majority of children with HIV infection are born to women from sub-Saharan Africa. ${ }^{911}$ Paediatric HIV infection is now a potentially preventable disease, and there is an urgent need to ensure that pregnant women from ethnic minority communities have information on the advantages and disadvantages of HIV testing appropriately presented by trained midwives.

\section{Clinical management}

TESTING

The diagnosis of HIV infection in a child has a devastating impact on a family. If the paediatrician suspects HIV infection, there is no place for surrogate testing (hypergammaglobulinaemia or CD4 count). The paediatrician should raise the possibility of HIV infection, which should then ideally proceed with the involvement of both parents, and a nurse/counsellor experienced in working with infected families. Testing is not urgent and can be delayed until the family are ready, and understand that a positive HIV test 
Table 2 Clinical categories of the paediatric HIV classification system (adapted from MMWR 1994;43:RR1-12)

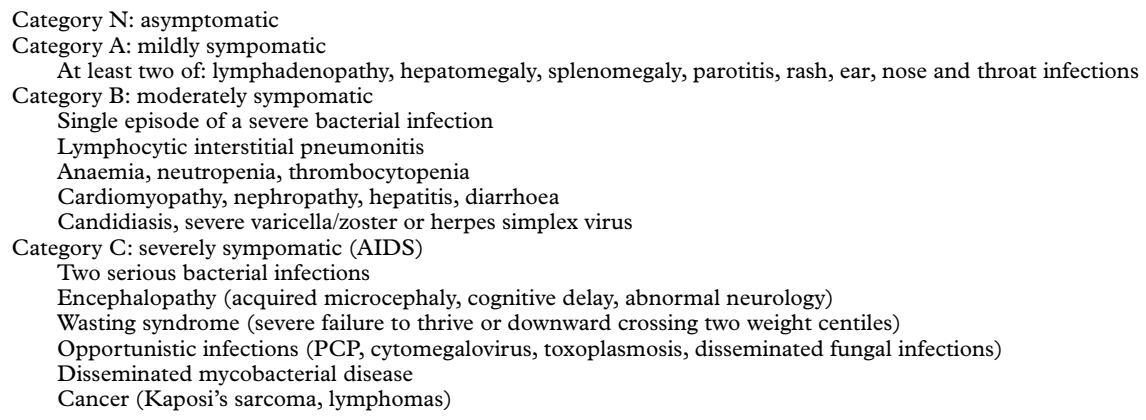

for the child means the mother, and possibly the father, will also be infected.

The early diagnosis of infection in children born to HIV positive mothers has improved with the introduction of more sensitive and specific diagnostic techniques of HIV detection in blood. Immune complex dissociated p24 antigenaemia is very specific after the first week of life, with high levels $(>100 \mathrm{pg} / \mathrm{ml})$ associated with a poor outcome. ${ }^{12}$ The polymerase chain reaction (PCR) has higher sensitivity, and a commercial kit (Roche Amplicor) is available. HIV culture remains the gold standard but is very expensive and not widely available. Any positive result must be confirmed on a separate blood sample. In the first week of life only about half of infected infants are positive by PCR with sensitivity rising to $95 \%$ by 1 month of age. ${ }^{13}$ Confirmation that a child is definitely not infected requires loss of maternal antibody which may take up to 18 months, but two negative tests by PCR or viral culture after 3 months of age suggests the child is not infected and Pneumocystis carinii prophylaxis may be stopped.

STAGING AND PROGNOSIS

The original 1987 Centers for Disease Control classification of paediatric HIV infection has been revised categorising children by the two parameters of clinical status and immune impairment. ${ }^{14}$ There are four clinical stages (N, A, B, C-for asymptomatic, mild, moderate, and severely symptomatic children) (table 2 ). The immune categories 1-3 represent increasing evidence of immune suppression using age related declining levels of the $T$ helper lymphocyte (CD4) count. A subgroup of vertically infected children have more rapid disease progression than adults. The European Collaborative Study has reported the prospective follow up of 124 vertically infected children. ${ }^{15}$ The majority of this cohort had received no antiretroviral treatment. An estimated 23\% (95\% confidence interval (CI) 15 to $31 \%$ ) of infected children developed AIDS in the first year of life, and nearly 40\% (95\% CI 27 to 50\%) by 4 years. Ten per cent had died by 1 year, and $28 \%$ by 5 years of age. Two years after an AIDS diagnosis $50 \%$ of the children were still alive. These data confirm other studies noting the poor outcome of the minority of infants diagnosed with AIDS in the first year of life usually with $P$ carinii pneumonia (PCP), cytomegalovirus, encephalopathy, or severe wasting - the rapid progressors - in contrast to older children presenting with bacterial infections or lymphocytic interstitial pneumonitis. ${ }^{16}{ }^{17}$ The reasons for this striking variation in outcome are still poorly understood. Rapid progression has been associated with the HLA-DR3 haplotype ${ }^{18}$ and TH2 cytokine production. ${ }^{19}$ It is also possible that rapid progression may represent intrauterine rather than peripartum HIV transmission. For many school age children however HIV infection is now a chronic disease, and there is increasing recognition of very long term survivors first presenting in late childhood and surviving into adolescence. ${ }^{20}$

FAMILY CLINICS

HIV infection is distinct from other chronic childhood illnesses because of the stigma and secrecy surrounding the diagnosis, with other family members usually infected. The model of care that has developed is the family clinic staffed by specialist paediatricians and adult physicians, with other multidisciplinary team members including nurse counsellors, psychologists, and dietitians. The clinic provides the focus for family centred care, minimising the hospital visits for families with poor health and often multiple social problems. Increasingly the model of shared care is developing between a specialist paediatric infectious diseases unit and the local paediatric unit with satellite clinics. Ideally the paediatrician will meet any HIV infected mother prenatally, and her infant will be followed up prospectively in the family clinic.

MEDICAL CARE

The emphasis for management of children with HIV is on the prevention, prompt recognition, and treatment of both infectious and organ disease. The primary immunisation course should be given including oral polio vaccine, although excretion of live virus may be prolonged, and parents should be warned to wash their hands carefully after changing nappies. Some paediatricians recommend inactivated polio vaccine. BCG should only be considered for uninfected infants born to HIV positive mothers from countries with high tuberculosis prevalence. Pneumovax should be given at 2 years of age. Influenza vaccine is probably not effective in children with symptomatic HIV disease.

If the mother has received perinatal AZT treatment, infants should continue to receive oral AZT for a total of 4-6 weeks, and then commence co-trimoxazole as prophylaxis against PCP, until the infant's HIV status has been determined. In the UK the majority of symptomatic children remain life long on co-trimoxazole as prophylaxis against PCP and bacterial infections.

A direct access policy should be encouraged for ill febrile children, with ward treatment protocols available for management of common problems. HIV infected children have a very high rate of pneumococcal septicaemia, and the early use of empiric intravenous antibiotics is required in sick febrile children (for example ceftriaxone). Prolonged courses of antibiotics at high doses may be necessary to eradicate infections. HIV related encephalopathy may present in young children with developmental delay, microcephaly, and spastic diplegia. The course may be rapidly progressive, or chronic with prolonged plateau periods. ${ }^{21}$ Milder cognitive failure with expressive language delay is seen more frequently in older children, and can be 
difficult to separate from the effects of parental illness, immigration, and poverty. Infected older children may require educational support for cognitive, language, and emotional/behavioural problems. ${ }^{22}$ Regular monitoring by a paediatric psychologist is very important for the early recognition of encephalopathy, monitoring the response to therapy, and responding to the psychosocial needs of the family. ${ }^{23}$

Growth faltering is a problem in symptomatic children. Any acute infection is associated with episodes of weight loss; in adults this is primarily related to reduced food intake. ${ }^{24} \mathrm{~A}$ paediatric dietitian should be involved in the care of the child, with the emphasis on encouraging a good diet, and facilitating catch up growth after acute infections. Single organ disease (for example cardiomyopathy, nephropathy), unexplained cognitive or growth failure, and cancers (for example lymphoma) may present as the first clinical problem in previously well school age children, ${ }^{20}$ emphasising the importance of HIV infection to all general paediatricians and paediatric subspecialists.

Antiretroviral treatment is changing rapidly, with the two recurrent questions being when to start treatment, and which drugs to use. New data suggest that there is very rapid production and destruction of CD4 cells and viral replication within asymptomatic HIV infected individuals. ${ }^{25}$ As more effective and better tolerated drug combinations become available it is likely that earlier intervention will be advocated. However because of the rapid emergence of resistance to many currently available drugs, and the often prolonged asymptomatic stage, paediatricians in the UK would generally start antiretroviral treatment only when the child becomes symptomatic, or there is a rapid fall in the CD4 count. The recently published Delta and ACTG 175 adult treatment trials both showed that for nucleoside naive patients combinations of AZT and didanosine or AZT and zalcitabine, were significantly more effective than AZT alone in delaying disease progression. The Concorde study demonstrated that early treatment with AZT alone conferred no major benefit over delaying treatment until progression of disease or worsening CD4 count. These studies have meant that adults starting antiretrovirals now routinely start with a combination of drugs. ${ }^{26}$ Triple therapy with the addition of either a protease inhibitor, or a non-nucleoside reverse transcriptase inhibitor is increasingly used, but long term clinical efficacy data are lacking. Interestingly for previously untreated children with mild to moderate symptoms the as yet unpublished ACTG 152 study demonstrated that both AZT/didanosine combination therapy and didanosine monotherapy appeared to be superior to AZT monotherapy. However because AZT has the best penetrance into the central nervous system, and in view of the established benefits of combination therapy in adults, most UK paediatricians will start symptomatic children on AZT and didanosine combination therapy (or AZT + zalcitabine if there is didanosine intolerance). Paediatricians in Europe, Canada, and South America are collaborating in a series of studies coordinated by the Paediatric European Network for the Treatment of Aids (PENTA). In the UK further information of current and planned treatment trials are available through the Medical Research Council HIV Clinical Trials Centre (tel: +44 (0) 171380 9991). A phase II study of adding lamivudine (3TC) to current treatment in symptomatic children (PENTA 4) has fully recruited. A phase II trial evaluating a new nucleoside analogue with a protease inhibitor in antiretroviral naive children (PENTA 5) is due to start in early 1997. Plasma HIV RNA levels are now recognised to be an important marker of disease progression in both adults and children, and it is currently recommended that the initiation and monitoring of antiretroviral treatment is based on clinical findings, CD4 counts, and viral load measurements. ${ }^{27}$ There is a problem in persuading some pharmaceutical companies to develop and licence paediatric formulations, and continued pressure is required to enable children to benefit from the rapid developments in antiretroviral treatment.

SOCIAL CARE

The diagnosis of HIV is initially often kept a secret within the immediate family reducing support from the extended family or friends. Respect of confidentiality is important, and families will often build close relationships with the 'HIV team'. ${ }^{23}$ Professional confidentiality can easily be confused with secrecy, and it is the responsibility of the health care worker to clarify that the professional exchange of clinically relevant information is in the best interest of the child and family. Increasing numbers of infected children are at school, underlining the need for universal precautions for blood spills in schools. It may be difficult to involve families with the statutory authorities. Social work departments face the difficult task of coordinating their delivery of services to deal confidentially with the complex multiple issues of housing and financial support, fostering, respite, and terminal care for both parents and children without families having to interact with a plethora of disconnected care workers. Families find it helpful to have one person coordinating services. There is an urgent need to improve practical help for families within the home, and to encourage the development of flexible respite and fostering care programmes. One of the principle difficulties for both families and health care workers is that of uncertainty, particularly related to the diagnosis in infancy, relationships within the family, future illnesses, and death. A report from Barnardos has estimated that at least 3000 children in the UK are now affected by HIV, the majority of whom are aged under $10 .^{28}$ Most of these children are not infected themselves with HIV, but have an infected parent. Some are being orphaned without knowing why their parents died. The most pressing of the unmet needs of these children as they enter adolescence is responding to the psychological burden they carry. ${ }^{29}$

\section{Conclusion}

North American family clinics are currently reporting that for over half their children one parent has died. Many children with HIV infection in the UK are multiply disadvantaged from the effects of poverty, racial discrimination, social isolation, family illness, and parental death. Parents do not want to talk to their children about HIV. Contrary to the spirit of the Children Act, the great majority of infected children do not know their diagnosis, causing legal and ethical dilemmas in the long term survivors. There is a steadily increasing group of children in the UK traumatised by HIV. With the rapid advances in drug treatment significantly improved survival with HIV is now a reality. There is hope that this will lead to wider community acceptance and openness for affected children.

In rich countries most perinatal HIV transmission is preventable, but this will require a more determined intervention in the teenage and pregnant populations. Paediatricians should have an advocacy role in promoting antenatal testing, developing services for affected families, and encouraging pharmaceutical companies to develop paediatric formulations of antiretroviral drugs. Recent evidence suggests that the experience of physicians in the management of AIDS is significantly associated with patient survival. ${ }^{30}$ In a time of contracting HIV budgets, purchasers of health care need to be aware of the projected requirement for increased paediatric HIV resources due to 
increasing numbers of children who will require more complex care as their disease advances.

World wide there is a particular need to develop cheap cost effective interventions for the vast majority of HIV infected children living in poverty in Africa and Asia; antiretroviral drugs will be far too expensive for the foreseeable future. Mass education campaigns can reduce but not eradicate the spread of HIV. The international public health priority is for a vaccine.

Paediatric Infectious Diseases Unit,

MIKE SHARLAND

5th Floor, Lanesborough Wing,

St George's Hospital,

Blackshaw Road,

London SW17 OQT

MRC HIV Clinical Trials Centre,

University College London Medical School,

London

DIANA GIBB

GARETH TUDOR-WILLIAMS

SAM WALTERS

Paediatric Infectious Diseases Unit, St Mary's Hospital,

London

Paediatric Infectious Diseases Unit,

VAS NOVELLI

Hospital for Sick Children,

London

See related articles on pages 330 and 334 .

1 Quinn TC. Global burden of the HIV pandemic. Lancet 1996:348:99-106.

2 Day NE. The incidence and prevalence of AIDS and prevalence of other Day NE. The incidence and prevalence of AIDS and prevalence of other
severe HIV disease in England and Wales for 1995 to 1999: projections severe HIV disease in England and Wales for 1995 to 1999
using data to the end of 1994. CDR Review 1996;6:R1-24.

3 Connor EM, Sperling RS, Gelber R, et al. Reduction of maternal-infant ransmission of human immunodeficiency virus type 1 with zidovudine treatment. $N$ Engl F Med 1994;331:1173-80

4 European Collaborative Study. Children born to women with HIV-1 infection: natural history and risk of transmission. Lancet 1991:337:253-60.

5 St Louis ME, Kamenga M, Brown C, et al. Risk for perinatal HIV-1 transmission according to maternal immunologic, virologic, and placental factors. FAMA 1993;269:2853-9.

6 Sperling RS, Shapiro DE, Coombs RW, et al. Maternal viral load, zidovudine treatment, and the risk of transmission of human immunodeficiency virus type 1 from mother to infant. $N$ Engl F Med 1996;335:1621-9.

7 Duliege A-M, Amos CI, Felton S, et al. Birth order, delivery route, and conDuliege A-M, Amos Cl, Fitton S, et al. Birth order, delivery route, and con-
cordance in the transmission of human immunodeficiency virus type 1 cordance in the transmission of human immuno
from mothers to twins. F Pediatr 1995;126:625-32.

\section{Legislation and drug trials}

Drugs used to treat children are often not licensed for use in this age group. Examples of licensed medicines used outside the conditions of the licence ('off label' usage) include the oral administration of a vitamin $\mathrm{K}$ injection preparation, and cisapride, which is commonly prescribed for infants, but is not licensed for children under 2 years of age. The main reason many drugs are not licensed for use in children is that drug trials, and in particular pharmacokinetic studies, are difficult to undertake in the young child. This leads to a reluctance on the part of pharmaceutical companies to become involved in such trials.

In Ireland, the Control of Clinical Trials and Drugs Act $1990^{1}$ has led to problems in paediatric research. In the United Kingdom, clinical trials in children are conducted with the consent of a local or hospital ethics committee. Before 1987, this was also the case in the Republic of Ireland. Following the death of a healthy adult subject in a trial, legislation was introduced to regulate clinical trials. Under this Act, trials of licensed drugs may be carried out
8 Dunn D, Newell ML, Ades A, et al. Risk of human immunodeficiency virus type 1 transmission through breast feeding. Lancet 1992;340:585-8.

9 Unlinked Anonymous HIV Surveys Steering Group. Unlinked anonymous HIV prevalence monitoring programme: England and Wales - data to the end of 1994. London: Department of Health, 1995

10 Dunn DT, Nicoll A, Holland FJ, et al. How much paediatric HIV infection could be prevented by antenatal screening. 7 Med Screening 1995;2:35-40.

11 Ades AE, Parker S, Cubitt D, et al. Two methods of assessing the risk-factor composition of the HIV-1 epidemic in heterosexual women: south east England, 1988-1991. Aids 1992;6:1031-6.

12 Arlievsky NZ, Pollack H, Rigaud M, Kaul A, Krasinski K, Borkowsky W. Shortened survival in infants vertically infected with human immunodeficiency virus with elevated p24 antigenaemia. F Pediatr 1995;127:538-43.

13 Rogers M, Schochetman G, Hoff R. Advances in the diagnosis of HIV infection in infants. In: Pizzo P, Wilfert C, eds. Pediatric AIDS: the challenge of HIV infection in infants, children and adolescents. 2nd Ed. Baltimore, of HIV infection in infants, children

14 Centers for Disease Control and Prevention. 1994 Revised classification for human immunodeficiency virus infection in children less than 13 years of age. $M M W R$ 1994;RR-12:1-10

15 The European Collaborative Study. Natural history of vertically acquired human immunodeficiency virus-1 infection. Pediatrics 1994;94:815-9.

16 Blanche S, Tardieu M, Duliege AM, et al. Longitudinal study of 94 symptomatic infants with perinatally acquired human immunodeficiency virus infection: evidence for a bimodal expression of clinical and biological symptoms. Am 7 Dis Child 1990;144:1210-5.

17 Tovo PA, DeMartino M, Gabiano C, et al. Prognostic factors and survival in children with perinatal HIV-1 infection. Lancet 1992;339:1249-53.

18 Just JJ, Abrams E, Louie LG, et al. Influence of host genotype on progression to acquired immunodeficiency syndrome among children infected with to acquired immunodeficiency syndrome among children infe
human immunodeficiency virus type 1.7 Pediatr 1995;127:544-8.

19 Vigano A, Principi N, Villa ML, et al. Immunological characterisation of children vertically infected with human immunodeficiency virus, with slow or rapid disease progression. F Pediatr 1995;126:368-73.

20 Grubman S, Gross E, Lerner-Weiss N, et al. Older children and adolescents living with perinatally acquired human immunodeficiency virus infection. Pediatrics 1995;95:657-63.

21 Lobato $\mathrm{MN}$, Caldwell $\mathrm{MB}, \mathrm{Ng} \mathrm{P}$, et al. Encephalopathy in children with perinatally acquired human immunodeficiency virus infection. $\mathcal{f}$ Pediatr 1995;126:710-5.

22 Papola P, Alvarez M, Cohen HJ. Developmental and service needs of school-age children with human immunodeficiency virus infection: a descriptive study. Pediatrics 1994;94:914-8.

23 Melvin D, Sherr L. The child in the family-responding to AIDS and HIV. Aids Care 1993;5:35-42.

24 Macallan DC, Noble C, Baldwin C, et al. Energy expenditure and wasting in human immunodeficiency virus infection. N Engl f Med 1995;333:83-8.

25 Perelson AS, Neumann AU, Markowitz M, et al. HIV-1 dynamics in vivo: virion clearance rate, infected cell life-span, and viral generation time. Science 1996;271:1582-6.

26 Pinching AJ. Managing HIV disease after Delta. BMf 1995;312:521-2.

27 Saag MS, Holodniy M, Kuitzkes DR, et al. HIV viral load markers in clinical practice. Nature Med 1996;2:625-9.

28 Imrie J, Coombes Y. No time to waste: the scale and dimensions of the problem of children affected by HIVIAIDS in the United Kingdom. Essex: Barnardos, 1995

29 Levine C. Orphans of the HIV epidemic: unmet needs in six US cities. Aids Care 1995;7:S57-S62.

30 Kitahata MM, Koepsell TD, Deyo RA, et al. Physicians experience with the acquired immunodeficiency syndrome as a factor in patient's survival. N Engl f Med 1996;334:701-6. with the approval of a recognised ethics committee, and the Irish Medicines Board. If, however, the aim of the trial is to study the effects of an unlicensed drug, or 'off label' use of a licensed drug - as is often the situation in studies involving children-application must first be made to the Minister for Health. The Minister, after consulting with the Irish Medicines Board, may approve, impose conditions, or refuse consent for the trial. If consent is approved, with or without conditions, the proposal must then be approved by the relevant ethics committee. The Act provides for trials of innovative treatment in minors who are ill. However, it prohibits any trial of treatment, where the aim is to prevent disease, if the subject is incapable of giving fully informed consent.

In contrast, we are permitted to administer substances, licensed or unlicensed, to a patient in the ordinary course of medical practice where the principal purpose is to prevent or treat disease. This does not require ethics committee approval, nor is it in the committee's remit to 